\title{
Comparison of Echo Reduction Techniques for One- Single Cut Antenna Measurements
}

\author{
Manuel José López Morales , Francesco Saccardi , Manuel Sierra Castañer , Lars J. Foged
}

\begin{abstract}
This paper presents a comparison between two different echo reduction techniques applied to one-single cut antenna measurements. These techniques are based on modal filtering and time filtering. The first technique uses a postprocessing of the far field one-single cut so as to obtain a modal expansion of cylindrical modes and to cancel the effect of spurious signals. The second one is based on filtering echoes out in time domain by applying a Fourier Transform to get a signal in which some spurious contributions can be filtered out. Such techniques are applied on the single cut measurements of a wideband horn (MVI SH2000) performed in a semi-anechoic chamber and on the measurements of a dipole (MVI SD1900) performed in a MVG spherical multi-probe system (StarLab). A smart combination of both methods is also applied in order to improve the results of the post processing with respect each different method.
\end{abstract}

Keywords - antenna measurement, semi-anechoic chamber, echo reduction techniques, time filtering, modal filtering, measurements, offset, single-cut measurements.

\section{INTRODUCTION}

During the last years, some techniques to reduce the effect of the echoes in near and far field have been presented. These echo reduction techniques make more accurate the measurements at lower frequencies or in outdoor environments, and could reduce the impact of towers, supporting structures, and so on.

Time-gating technique is the most classical option. The main idea is to separate the direct signal from the reflections, employing a time-domain representation. In the initial approaches [1], [2], the measurement was directly performed in the time-domain. However, the complexity of the equipment is lower using frequency-domain measurements as it is proposed in [3]. From such measurements, the time response is immediately calculated applying an inverse Fourier transform (IFT). Once reflections have been gated out, the signal is converted to frequency-domain by means of a Fourier transform (FT). The fast algorithms used to compute the FT and IFT require a constant frequency step.

The authors want to acknowledge the COST VISTA project for financing the short stays of UPM students in Pomezia, the Madrid Region Government project for financing the project Space Debris Radar (S2013/ICE-3000 SPADERADAR-CM) and the Spanish Government, for the support of the project ENABLING5G "Enabling Innovative Radio Technologies for $5 \mathrm{G}$ networks" (TEC2014-55735-C3-1-R)
So, when there are frequency regions where the field varies quickly, a small step has to be employed in all the bandwidth to get a good characterization in those regions, and therefore the measured time may increase considerably. One technique based on digital signal processing was proposed in [4] to obtain the time response also from frequency-domain measurements, but not necessarily with uniform sampling.

Other solutions are the compensation methods which can be applied to avoid measuring the Antenna Under Test (AUT) several times at different distances to the probe. The most well-known approach is the test zone field compensation [5]. Its principal drawback is the computational complexity which is reduced in [6].

Other possible approaches are the frequency decomposition techniques. Initial approaches are shown in [7] and [8]. A variation of the previous approaches is the pencil-of-function method [9]. Previous methods have different drawbacks, which can be solved employing the pencil-of-function method or its improved version, the matrix pencil method, [10]-[13].

Other approaches based on spherical or cylindrical mode filtering or in spatial filtering appeared during the last years. A technique named MARS [14]-[17] has also been proposed to cancel undesired reflected signals. It is based on a special filtering of unwanted cylindrical modes [14] or spherical modes [15]. This technique has been extended to planar and far field measurements [16]-[17]. Something similar is used by ISOFIL TER [18].

The last group of algorithms is based on spatial filtering technique. The basis of the method is a diagnostic technique whose aim is to provide the extremely near-field of the AUT. In [19] diagnosis based on FFT is applied to cancel out the reflections, and the algorithm was applied to Planar Near Field and Cylindrical Near Field Systems. An extension to Far Field systems was presented in [20].

An innovative approach for echo reduction based on using a multi-probe system so that enough information is available to resolve the direct contributions of the antenna under test and the multipath signals has been presented in [21].

This paper has the main objective of analyzing two echo reduction techniques: the modal filtering and the time domain filtering, both applied to $\mathrm{FF}$ one single cut measurements. A 
combination of both techniques is also studied in order to assess the further improvements that can be obtained. The new contributions of this paper are the analysis of the application of modal filtering technique to one single cut measurements and the combination of time gating and modal filtering.

The paper is divided in the following sections. Section 2 briefly details the theory behind each method. Section 3 shows some results obtained after applying each method to different antennas and set-ups. Section 4 explains how to combine both methods smartly and presents some results of this combination. Finally, section 5 concludes the paper.

\section{ECHO REDUCTION THEORY}

This section presents a brief overview of each method studied: modal filtering and time filtering. The post-processing techniques are applied directly to far field data which can be measured directly if the distance is large enough or they can be obtained from the NF with a NF/FF transform applied to classical scanning geometries (spherical, planar or cylindrical [22]). In [23] it has been also presented an innovative NF/FF transformation technique that allows performing an accurate evaluation of the FF starting from a single cut NF. Such methodology is very interesting as it allows to quickly measure the main radiation characteristic of an antenna avoiding the acquisition of the full 3D dataset.

\section{A. Modal filtering}

Modal filtering is performed transforming the near or far field pattern to spherical or cylindrical spectrum and turning off modes associated to echo contributions. In this paper the transformation is applied from far field to cylindrical waves of a single cut measurement. It is well known that the cylindrical wave spectrum is bounded according to the antenna under test (AUT) geometry in the cut direction (equations (1) and (2)).

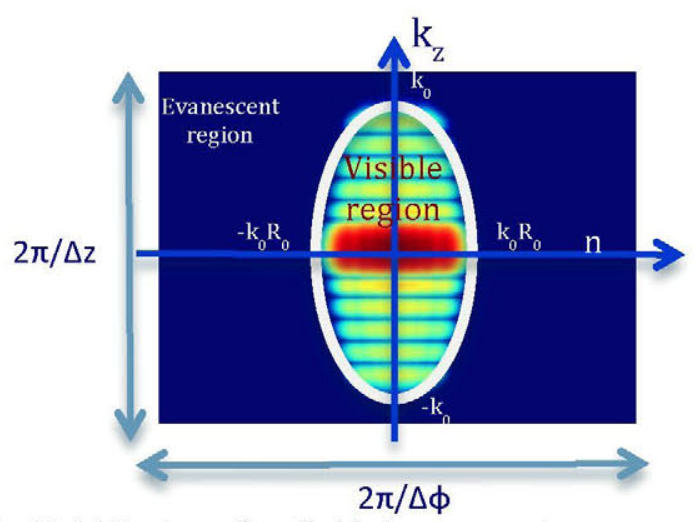

Fig. 1. Modal Spectrum of a cylindrical measurement.

$$
\begin{gathered}
n^{2}+\left(k_{z} R_{0}\right)^{2}<\left(k_{o} R_{0}\right)^{2} \\
N_{\max }=k_{0} R_{o}+n_{1}
\end{gathered}
$$

In (1) $R_{0}$ is the minimum radius of the cylinder fully inclosing the AUT. Fig. 1 shows cylindrical modes for a full cylinder measurement. In far field, the cylindrical modes can be simply obtained through a FFT of the radiation pattern. This can be deducted from (3), where for each $\theta$ value, $a_{n}$ and $b_{n}$ correspond to the FFT of the far field pattern (the other terms are just constants or phase shifters that are not necessary to be considered for filtering purposes). After filering in the spectral domain, just an IFFT is necessary to come back to the spatial domain.

$$
\overrightarrow{E_{f a r}}(r, \theta, \phi)=-2 k_{0} \sin (\theta) \frac{e^{-j k_{0} r}}{r} \sum_{n=-\infty}^{\infty}\left(j^{n}\left(a_{n}\left(k_{z}\right)\right) \widehat{\emptyset}+j b_{n}\left(k_{z}\right) \hat{\theta}\right) e^{j n \emptyset}(3)
$$

\section{B. Time filtering}

Time filtering was the first method used for echo reduction. The concept about this method has been explained in the introduction of this paper. In [24], this technique has been applied to the NF of the antenna. In this paper is instead applied on the FF of the AUT, where direct ray and interferences can still clearly be detected because the transformation from $\mathrm{NF}$ to $\mathrm{FF}$ is just a linear operation, which does not impact the effectiveness of this filtering method. The main drawback is the number of samples and the required frequency band to have enough resolution in time domain.

\section{APPLICATION OF THE METHODS TO SINGLE CUT MEASUREMENT}

In this section results obtained with each method are shown. Two different antennas are measured in two different set-ups. In the first case, a horn antenna is measured in a classical spherical near field configuration. In the second case a dipole antenna is placed in a multi-probe measurement system.

\section{A. Measurement Results for the Wideband Horn Antenna}

The MVI SH2000 horn antenna is measured in a single cut near field set-up able to perform single cut data rotating on azimuth (Fig. 2). In this case, the AUT is offset $50 \mathrm{~cm}$ from the origin of the measurement system. Before applying any processing, the AUT is mathematically translated to the origin. To add a reflection to be reduced later by a post processing, a metallic plate was collocated into the chamber. It is also important to say that different windows have been used in each domain to filter echoes out, obtaining best results with Hanning or rectangular window. The important issue in modal domain was choosing a proper window width. In time domain there was a big difference between applying different windows. A rectangular window in time domain usually creates a big Gibbs effect because of truncation error, while a proper Hanning window mitigates this error.

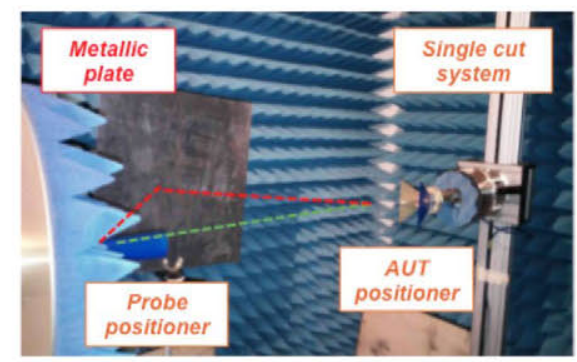

Fig. 2. Single cut measurements setup for the SH2000 wideband horn. 
Fig. 3 shows the effect of the modal filtering for this particular case. It is observed that the contribution from the echo can be perfectly filtered since it is enough separated from the contribution of the AUT. This is due to the offset of the AUT, as it will be observed in the photograph of the setup.

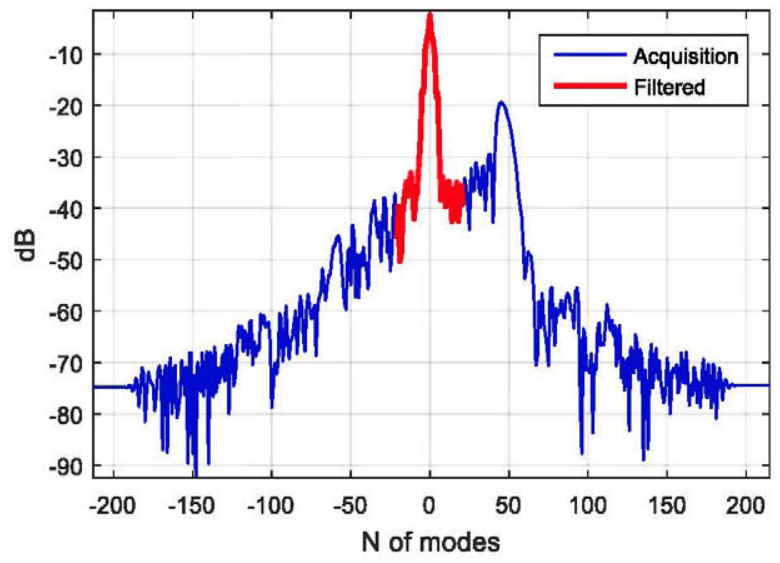

Fig. 3. Single cut spectral domain of the Wideband Horn (SH2000). Measuement is performed with a reflecting plate.

With the measurements performed, this method allows us to obtain a great agreement between the reference pattern and the pattern with the reflection after filtering (Fig. 9). The red pattern is the reference one, the blue one contains the effects of the reflection and, finally, the green one is the pattern after modal filtering. Instead, when time gating is applied to this specific set-up, it is very difficult to obtain accurate results. Fig. 4 also shows the signal in time domain. It is observed that the direct ray and the contribution from the echo are very close. Fig. 9 also shows the results for the pattern after time filtering for the central frequency $(8 \mathrm{GHz})$. The cyan pattern shows the results after time filtering is applied. However, Fig. 5 shows the results for the extreme value of the measured band ( $6 \mathrm{GHz})$. In this case, it is observed that the gate is not properly adjusted, and the radiation pattern is not good enough. This could be solved by increasing the measurement bandwidth, but sometimes this is not possible.

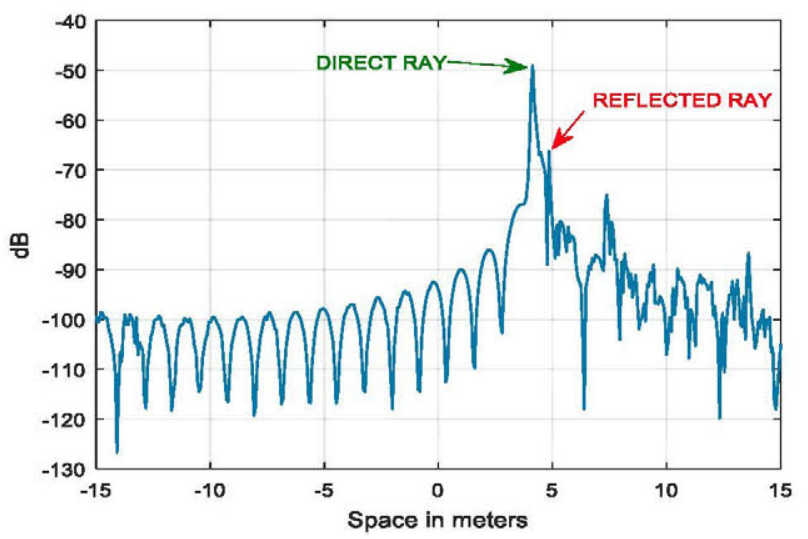

Fig. 4. Time domain signal for the SH2000 antenna.

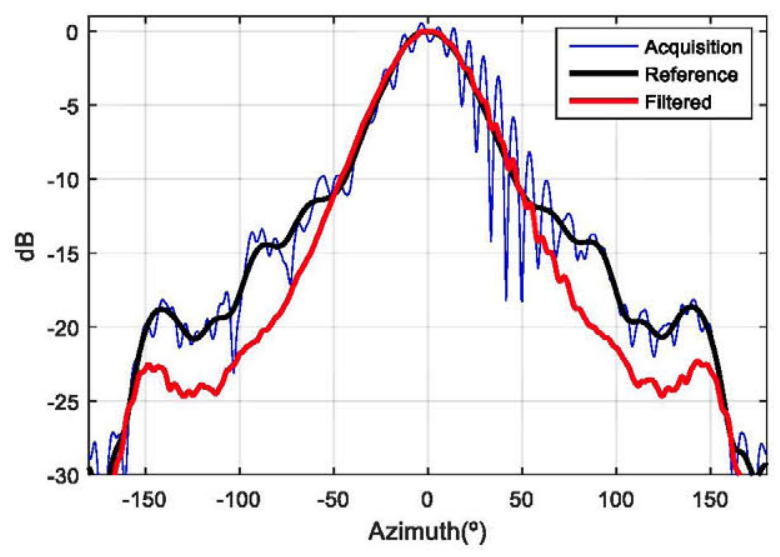

Fig. 5. Radiation patterns of the $\mathrm{SH} 2000$ (a) $6 \mathrm{GHz}$ before and after time filtering.

\section{B. Measurement Results for the Dipole Antenna}

The second configuration is a dipole antenna measured in a multi-probe spherical measurement system (MVG StarLab System). Again, a large metallic plate (square of $50 \mathrm{~cm}$ ) has been placed to create a large echo in the measurement (Fig. 6). The antenna under test is the MVI SD1900, a dipole antenna whose central frequency is $1900 \mathrm{MHz}$. Time gating has been used from measurements in the frequency band from 1.7 to 2.2 $\mathrm{GHz}$ with a step of $10 \mathrm{MHz}$. Modal filtering has been applied just to the central frequency $(1.9 \mathrm{GHz})$.

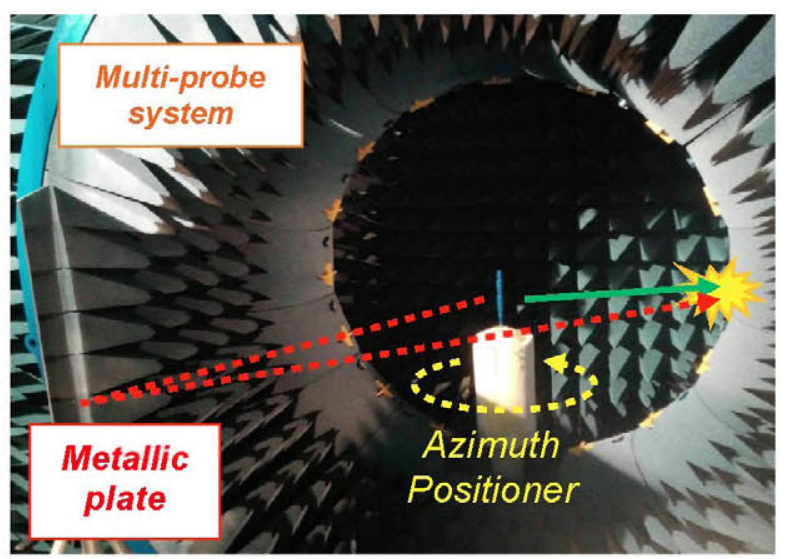

Fig. 6. SD1900 in the StarLab multi-probe system.

In Fig. 7 it is observed that the contribution from the echo cannot easily be filtered out, some high mode echoes can be filtered out, but this is not the case for lower ones. This is due to the fact that, since the AUT is centered, the modal coefficients of the echoes cannot be separated from the modal coefficients from the AUT (both are centered). Fig. 10 and Fig. 11 show the radiation pattern for the horizontal and vertical plane respectively. In both cases the blue pattern is the measurement with reflection, the red pattern is the reference and the green pattern shows the results after applying modal filtering. It is observed that some improvement is obtained in the vertical plane but almost none in the horizontal one. This is due to the fact that, since the horizontal plane is measured 
rotating the AUT, it is not possible to well represent the echo signal in the spectral domain and thus filter it out.

Instead, time gating works pretty well in this case. Fig. 8 shows the signal in time domain. Clearly, direct ray and echo can be detected and echo can be filtered out. Again, Fig. 10 and Fig. 11 show the radiation pattern in both planes: the results are pretty good in this situation.

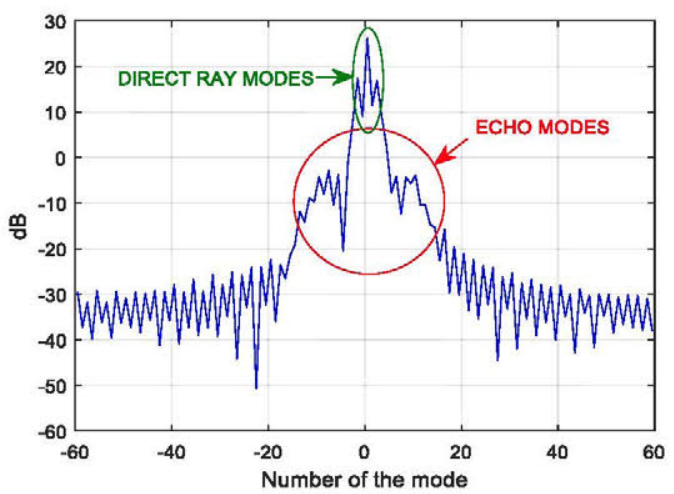

Fig. 7. Spectral domain of the SD1900 for one single cut elevation.

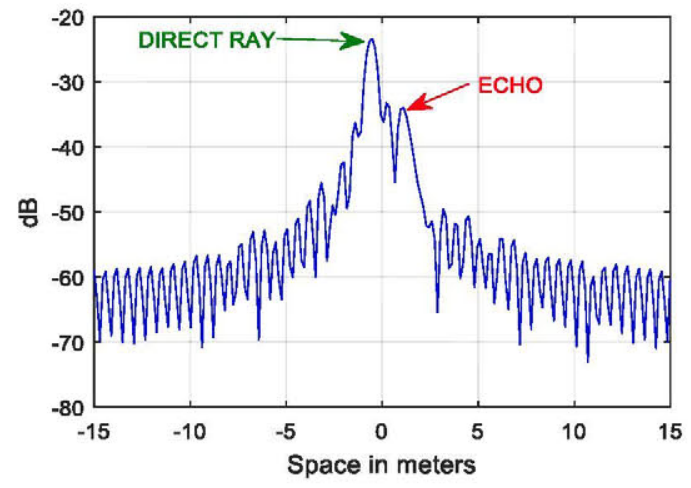

Fig. 8. Time Domain Signal of the SD1900 for the point $\theta=90, \phi=180$.

\section{COMBINATION OF METHODS}

The previous section showed that modal filtering is the best option for one setup and time gating for the other one. Both methods work in a different way, each one with its own advantages and limitations. For modal filtering, an offset in the AUT with respect the center of coordinates of the measurement system is required if the AUT is moving so as to properly separate direct and reflected ray. On the other side, in the case of the time gating, a wide band acquisition is required to filter out contributions that are not separated enough in time. Sometimes, for very close rays, even enough bandwidth will not be enough, and those rays may not be separated. As well as this, for both cases, the use of very narrow windows would destroy some important information and would also be very difficult to be adjusted. By using a combination, windows specifications can be less restrictive, having a process less sensitive to errors and easier to be automatized, because so much adjustment is not necessary. With this, the error reduction is extended to more cases. Both methods have a low computational load, so the combination will not be computationally restrictive.

In this paper, this combination is applied to one-single-cut measurements, but it can be easily extrapolated to other situations. In fact, the procedure uses the advantages of applying both techniques on the far field cut. In the case of modal filtering, the single-cut cylindrical modes are obtained from each individual cut, allowing a filtering in this domain. In the case of time filtering, the frequency domain to time domain transformation is also performed in far field domain. This is done after a phase shift of the results to the centre of the AUT.

It is important to emphasize that this method is not a "cureall" method, because, as can be observed for each situation commented, if one method on its own does not improve results with reflections, the combination will not either. In the dipole situation there is an offset limitation, and in the SH2000 very close rays limit the algorithm efficiency. For the dipole situation it is clearer than the combination improves results, because time filtering removes echo contribution, and modal filtering removes other causes of inaccuracies such as the antenna positioner, for instance.

The results of the combination are shown in Fig. 9, 10 and 11 , with the black pattern.

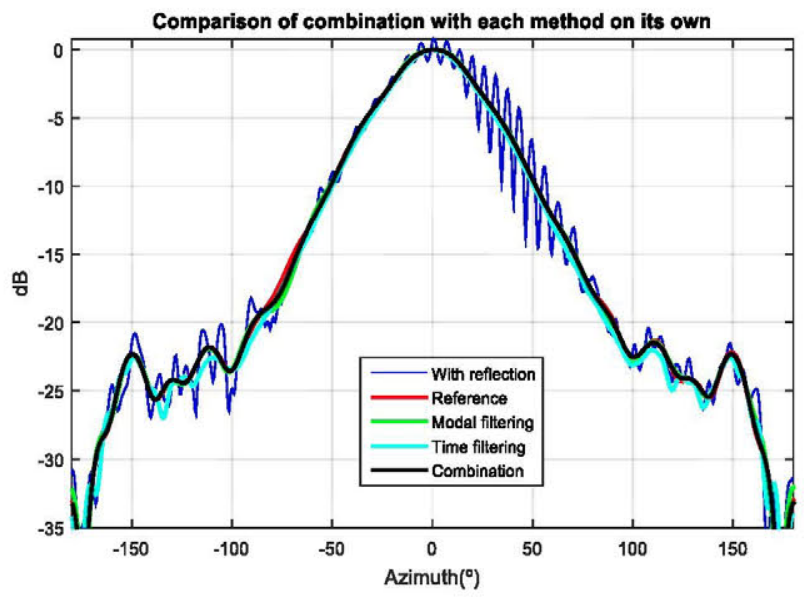

Fig. 9. Comparison between different echo reduction technique for the azimuth cut measurement of the $\mathrm{SH} 2000$.

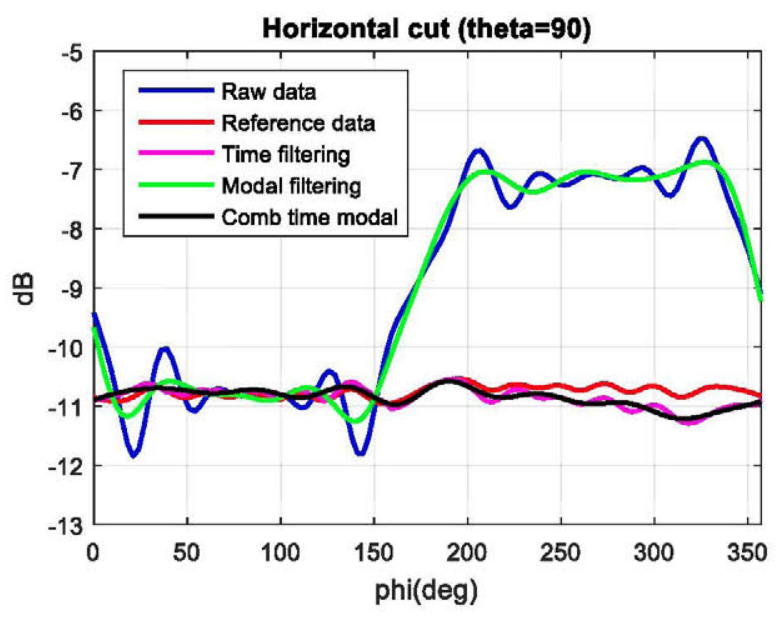

Fig. 10. Comparison between different echo reduction technique for the horizontal cut measurement of the SD1900 dipole. 


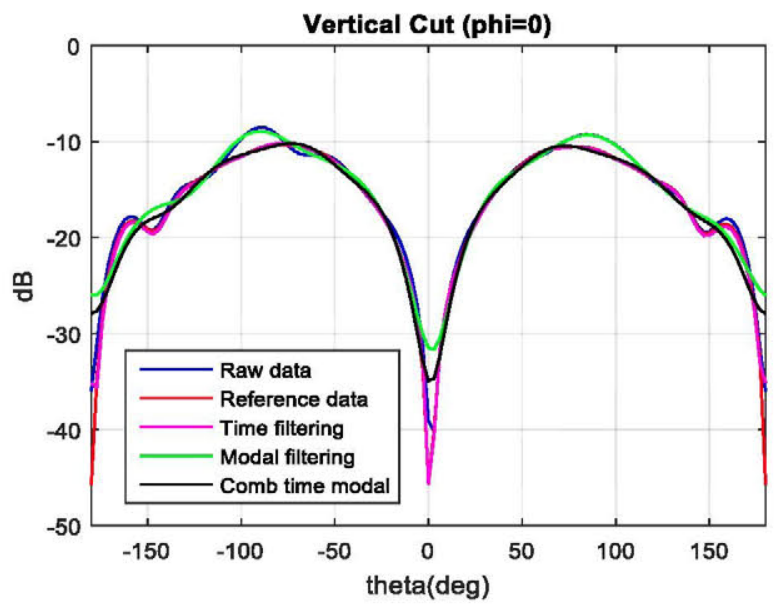

Fig. 11. Comparison between different echo reduction technique for the vertical cut measurement of the SD1900 dipole.

\section{CONCLUSIONS}

This paper showed the application of two different echo reduction techniques for two different AUT and measurement setups. Echo reduction techniques have been applied to single cut far field pattern obtained from NF with a NF/FF transformation. The algorithms can in fact be applied to specific cuts where the user could detect problems or to single cuts NF measurements. In fact, this algorithm can be combined with the single cut NF/FF transformation software presented in previous work [24].

Time gating can be equally applied to both the NF and FF since the NF/FF transform is a linear operation. Therefore, the results are the same because even if the FF is supposed to be at infinite distance (the relative distance between echo and direct field remains constant). In the case of modal filtering, cylindrical modes were used since there is a relation between the mode coefficients and the single cut FF.

For each case, there are improvements, but in one case the improvement s are more appreciable using modal filtering and in the other case using time gating. This strongly depends on the measurement setup and the relative position of the source of echo. In any case, it was shown that the combination of both techniques can be applied with good performance.

\section{REFERENCES}

[1] J. D. Young, D. E. Svoboda, and W. D. Burnside, "A comparison of time- and frequency-domain measurement techniques in antenna theory," IEEE Trans. Antennas Propagat., vol. 21, No. 4, pp. 581-583, Jul., 1973.

[2] G. A. Burrell and A. R. Jamieson, "Antenna radiation pattern measurement using time-to-frequency transformation (TFT) techniques," IEEE Trans. Antennas Propagat., vol. 21, No. 5, pp. 702-704, Sep., 1973.

[3] S. Loredo, M. R. Pino, F. Las-Heras, and T. K. Sarkar, "Echo identification and cancellation techniques for antenna measurement in non-anechoic test sites," IEEE Antennas Propagat. Mag., vol. 46, No. 1, pp. 100-107, Feb., 2004.

[4] M. Dadić and R. Zentner, "A technique for elimination of reflected rays from antenna measurements performed in echoic environment," $A E \ddot{U}$ Int. J. of Electron. Commun., vol. 61, No. 2, pp. 90-94, Feb. 2007.

[5] D. N. Black and E. B. Joy, "Test zone field compensation," IEEE Trans. Antennas Propagat., vol. 43, No. 4, pp. 362-368, Apr, 1995.
[6] D. A. Leatherwood, E. B. Joy, "Plane wave, pattern subtraction, range compensation," IEEE Trans. Antennas Propagat., vol. 49, No. 12, pp. 1843-1851, Dec, 2001.

[7] M. L. Van Blaricum and R. Mittra, "A technique for extracting the poles and residues of a system directly from its transient response," IEEE Trans. Antennas Propagat., vol. AP-23, No. 6, pp. 777-781, Nov., 1975.

[8] T. K. Sarkar and O. Pereira, "Using the matrix pencil method to estimate the parameters of a sum of complex exponentials," IEEE Antennas Propagat. Mag., vol. 37, No. 1, pp. 48-55, Feb., 1995.

[9] T. K. Sarkar, J. Nebat, D. D. Weiner, and V. K. Jain, "Suboptimal approximation/identification of transient waveforms from electromagnetic systems by pencil-of-function method," IEEE Trans. Antennas Propagat., vol. AP-28, No. 6, pp. 928-933, Nov., 1980.

[10] Y. Hua and T. K. Sarkar, "Generalized pencil-of-function method for extracting poles of an EM system from its transient response," IEEE Trans. Antennas Propagat., vol. 37, No. 2, pp. 229-234, Feb., 1989.

[11] R. S. Adve, T. K. Sarkar, O. M. C. Pereira-Filho, and S. M. Rao, "Extrapolation of time.domain responses from three-dimensional conducting objects utilizing the matrix pencil technique," IEEE Trans. Antennas Propagat., vol. 45, No. 1, pp. 147-156, Jan., 1997.

[12] B. Fourestié, Z. Altman, J. Wiart, and A. Azoulay, "On the use of the matrix-pencil method to correlate measurements at different test sites," IEEE Trans. Antennas Propagat., vol. 47, No. 10, pp. 1569-1573, Oct., 1999.

[13] B. Fourestié and Z. Altman, "Gabor schemes for analyzing antenna measurements," IEEE Trans. Antennas Propagat., vol. 49, No. 9, pp. 1245-1253, Sep., 2001.

[14] S. Gregson, A. Newell, and G. Hindman, "Reflection suppression in cylindrical near-field antenna measurement systems-cylindrical MARS," in Antenna Meaurement. Techniques Assoc., AMTA 2009, Salt Lake City, UT, November 1-6, pp. 119-125.

[15] G. Hindman and A. Newell, "Reflection suppression in large spherical near-field range," in Antenna Meaurement. Techniques Assoc.. AMTA 2005, Newport, RI, October 30-November 4, pp. 270-275.

[16] S.F. Gregson, A.C. Newell, G.E. Hindman, M.J. Carey, "Application of Mathematical Absorber Reflection Suppression to Planar Near-field Antenna Measurements", 5th European Conference on Antennas and Propagation, Rome, 11th -- 15th April, 2011.

[17] S.F. Gregson, A.C. Newell, G.E. Hindman, M.J. Carey, "Advances in Cylindrical Mathematical Absorber Reflection Suppression", $4^{\text {th }}$ European Conference on Antennas and Propagation, Barcelona, 12th16th April, 2010.

[18] D. Hess. "The IsoFilter ${ }^{\mathrm{TM}}$ Technique Isolating and Individual Radiator from Spherical Near-Field Data Measured in a Contaminated Environment". Proceedings of the European Conference on Antennas and Propagation 2007.

[19] F.J. Cano-Fácila, S. Burgos, F. Martín, M. Sierra-Castañer, "New Reflection Suppression Method in Antenna Measurrement Systems Based on Diagnostic Techniques". IEEE Transactions on Antennas and Propagation, vol. 59, no. 3, March 2011, pp. 941-949.

[20] M. Sierra-Castañer, F. Cano-Fácila, L.J. Foged, F. Saccardi, G. Nader Kawassaki, L. dos Reis Raimundi, S. A. Vilela Rezende. "A Reflection Suppression Technique for Far Field Antenna Measurements". Proceedings of the EuCAP 2013.

[21] T K. Yinusa and T. Eibert, "A multi-probe antenna measurement technique with echo suppression capability," IEEE Trans. Antennas Propag., vol. 61, no. 10, pp. 5008-5016, Oct. 2013

[22] A. Yaghjian, "An Overview of Near-Field Antenna Measurements," IEEE Trans. Antennas Propagat., vol. 34, no. 1, pp. 30-45, Jan. 1986

[23] T. Salmerón-Ruiz, M. Sierra-Castañer, F. Saccardi, S. Burgos, F. J. Cano-Fácila, Lars J. Foged. "A Fast Single Cut Spherical Near-Field-toFar-Field Transformation Using Cylindrical Modes". Proceedings of 8th European Conference on Antennas and Propagation, EuCAP 2014. Den Haag (Netherlands). April 2014.

[24] M. Sierra Castañer, P. González-Blanco García, M.J. López Morales, F. Saccardi, L. J. Foged. "Time and Spatial Filtering for Echo Reduction in Antenna Measurements". Proceedings of the AMTA Symposium 2015. Long Beach, CA, United States. October 2015. 